\title{
Computation of Zagreb Indices on $k$-Gamma Graph
}

\author{
G. Kiruthika ${ }^{1}$, Dr. D. Kalamani ${ }^{2}$ \\ ${ }^{I}$ Research Scholar, Department of Mathematics, Bharathiyar \\ University PG Extension \& Research Centre, Erode, India \\ ${ }^{2}$ Associate Professor, Department of Mathematics, Bharathiyar \\ University PG Extension \& Research Centre, Erode, India \\ Email:aisswaryakiruthika@gmail.com ${ }^{1}$,kalamanikec@gmail.com ${ }^{2}$
}

\begin{abstract}
In this paper, we newly define Gamma Graph $(\Gamma), k$-Gamma Graph $\left(\Gamma_{k}\right)$ for every $k \geq 2$ and junction vertex by using elementary graph operation. Another important graph called Swastika Graph $(S W)$ is also defined. Additionally we shall give generalization result for $n$-Swastika $\left(S W_{n}\right)$ for any $n \geq 1$. We shall derive Line Graph for $k$-Gamma Graph $\left(\Gamma_{k}\right)$ and $n$-Swastika $\operatorname{Graph}\left(S W_{n}\right)$. Then we shall calculate some of topological indices namely Third Zagreb Index, First and Second Multiple Zagreb index, First and Second Zagreb polynomials for $k$-Gamma $\left(\Gamma_{k}\right), k$-Gamma Line $\operatorname{Graph}\left(\Gamma_{k}\right)$ where $k \geq 2, n$-Swastika Graph $\left(S W_{n}\right)$ and $n$-Swastika Line Graph $L\left(S W_{n}\right)$ for any $n \geq 1$.
\end{abstract}

Index Terms-k-Gamma Graph $\left(\boldsymbol{\Gamma}_{\boldsymbol{k}}\right)$, Swastika $\operatorname{Graph}\left(\boldsymbol{S} \boldsymbol{W}_{\boldsymbol{n}}\right)$, Junction Vertex, Zagreb Indices.

\section{INTRODUCTION}

In Chemical Graph Theory, molecular graphs represent the molecular structure of a certain chemical compound and it is associated with different properties which are found in molecular graph connectivity with the corresponding chemical compounds.

A graph recognizes the easiest way of approach to understand its excellence, by means of numeric constant. Topological descriptors are based on the graph impression of the molecule and can also encode chemical information concerning atom type and bond multiplicity. The molecular descriptors is the final result of logic and the mathematical procedure which convert the chemical information fixed with in a symbolic representation of molecule in to a useful number.

The molecular structure of a drug can be considered as graph by suppressing the hydrogen atoms. The topological index of molecular graph is helpful to find properties of the drug. The drugs can be classified by using its properties. Some of the methods used for classification are Quantitative Structure Activity Relationship (QSAR) and Quantitative Structure Property Relation (QSPR) and Fuzzy Lattice Neural Networks (FLNN) etc., Thus, the topological indices are very essential tool in the field of Nanotechnology, Drug Discovery, Computer Networks, Designing Tool, Information Technology etc.,

The current researches about topological descriptors are used to qualify different chemical properties of drugs. Since, topological indices on special kinds of various graphs have been found previously which is based on the degrees of vertex and edge respectively. In this paper, we motivated to investigate a new set of graphs such as k-Gamma Graph, k-Gamma Line
Graph, Swastika Graph, n-Swastika Graph, n-Swastika Line Graph. It brings our curiosity to find some chemically interesting graphs.

\section{PRELIMINARY RESULTS}

Some of the well-known topological indices are defined for the simple graph $\mathrm{G}$.

Definition 2.1. The Third Zagreb index [3] of a graph $\mathrm{G}$, denoted by $M_{3}(G)$, is defined as

$$
M_{3}(G)=\sum_{u v \in E(G)}|d(u)-d(v)|
$$

Definition 2.2. The First Multiple Zagreb index [5] of a graph $\mathrm{G}$, denoted by $P M_{1}(G)$, is defined as

$$
P M_{1}(G)=\prod_{u v \in E(G)}[d(u)+d(v)]
$$

Definition 2.3. The Second Multiple Zagreb index [5] of a graph $\mathrm{G}$, denoted by $P M_{2}(G)$, is defined as

$$
P M_{2}(G)=\prod_{u v \in E(G)} d(u) d(v)
$$

Definition 2.4. The First Zagreb Polynomial index [6] of a graph $\mathrm{G}$, denoted by $M_{1}(G, x)$, is defined as

$$
M_{1}(G, x)=\sum_{u v \in E(G)} x^{[d(u)+d(v)]}
$$




\section{Available online at www.ijrat.org}

Definition 2.5. The Second Zagreb Polynomial index [6] of a graph $\mathrm{G}$, denoted by $M_{2}(G, x)$, is defined as

$$
M_{2}(G, x)=\sum_{u v \in E(G)} x^{d(u) d(v)}
$$

where $d(u)$ and $d(v)$ represent the degrees of vertices of $u$ and $v$ in the graph $\mathrm{G}$ respectively.

Definition 2.6. The Line Graph $[1,4,7]$ of a graph $G$ is the graph $L(G)$ with the edges $\mathrm{G}$ as its vertices, and where two edges of $\mathrm{G}$ are adjacent in $L(G)$ if and if they are incident in $\mathrm{G}$.

Definition 2.7. An Edge Contraction [9] is an operation which removes an edge from a graph while simultaneously merging the two vertices that it is previously joined.

The above definitions are the basic definitions which are useful in subsequent sections. The computation of topological indices of various graph families may be referred in [1, 4, 6 and 8].

\section{SOME ZABREB INDICES ON $\boldsymbol{k}$-GAMMA GRAPH AND ITS LINE GRAPH}

In this section, we shall used to calculate the some of the topological indices which are defined earlier for our newly defined $k$ - Gamma Graph and its Line Graph.

Definition 3.1. An undirected graph having three vertices and its two adjacent edges, in which edges are perpendicular is called Gamma Graph. It is denoted by $\Gamma$.

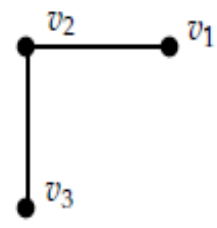

Fig. 3.1. Gamma Graph

From the above Fig.3.1. we have, $v_{1}=$ starting vertex, $v_{2}=$ incident vertex, $v_{3}=$ end vertex. Then $v_{1}$ and $v_{3}$ are pendant vertices and deg $v_{2}=2$.

Example 3.1. The Bent molecule like Silicon-diOxide $\left(\mathrm{SiO}_{2}\right)$ is a Gamma Graph. It helps to find the antibacterial activity.
Definition 3.2. If $k$ - number of Gamma Graphs shares a common vertex (starting vertex), then the graph is said to be $k$ - Gamma Graph and is denoted by $\Gamma_{k}$, where $k \geq 2$.

Definition 3.3. The common vertex in $k$ - Gamma Graph is called Junction vertex. The angles between any two adjacent Gamma Graphs are equal. If $\theta$ is the angle between any two adjacency Gamma Graphs then $\theta=\frac{360}{k}$, where $k \geq 2$. The vertex set and edge set of $k$-Gamma Graph $\Gamma_{k}$ is given by $|V|=2 k+1$ and $|E|=2 k$.

Theorem 3.1. Let $\Gamma_{k}$ be the $k$-Gamma Graph, where $k \geq 2$. Then its topological indices are given by

(1) $M_{3}\left(\Gamma_{k}\right)=2 E_{2}\left(L\left(\Gamma_{k}\right)\right)$

(2) $P M_{1}\left(\Gamma_{k}\right)=3^{k}(2+k)^{k}$

(3) $M_{2}\left(\Gamma_{k}\right)=E\left(\Gamma_{k}\right)^{E\left(\Gamma_{k}\right)}$

(4) $M_{1}\left(\Gamma_{k}, x\right)=k x^{2}\left(x+x^{k}\right)$

(5) $M_{2}\left(\Gamma_{k}, x\right)=k\left(x^{2}+x^{2 k}\right)$

Proof.

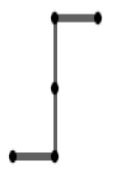

$k=2$

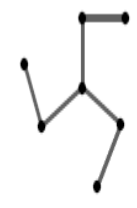

$k=3$

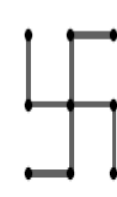

$k=4$

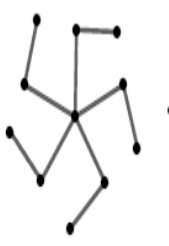

$k=5$
Fig 3.2. $k$ - Gamma Graph $\left(\Gamma_{k}\right)$

Consider the $k$-Gamma $\operatorname{Graph}\left(\Gamma_{k}\right)$, for all $k \geq 2$. From the Fig. 3.2., the number of vertices and edges of $\Gamma_{k}$ is given by $2 k+1$ and $2 k$ respectively. Then the vertex set can be divided into $k$ vertices of degree one, $k$ vertices of degree two, and one vertex of degree $k$. The edge set $E\left(\Gamma_{k}\right)$ can be divided into two edge partitions based on the degrees of its end vertices. The first partition of edges $E_{1}\left(\Gamma_{k}\right)$ contains $k$ edges $u v$, where $d_{\Gamma_{k}}(u)=1, d_{\Gamma_{k}}(v)=2$ and the second partition of edges $E_{2}\left(\Gamma_{k}\right)$ contains $k$ edges $u v$, where $d_{\Gamma_{k}}(u)=2, d_{\Gamma_{k}}(v)=k$. Here $d_{\Gamma_{k}}(u)$ and $d_{\Gamma_{k}}(v)$ are denoted as the degrees of its end vertices respectively. Then, we shall obtain our required results for the graph $\left(\Gamma_{k}\right)$ as follows,

(1) The Third Zagreb index

$$
\begin{gathered}
M_{3}\left(\Gamma_{k}\right)=\sum_{u v \in E\left(\Gamma_{k}\right)}|d(u)-d(v)| \\
=k(k-1) \\
=2 E_{2}\left(L\left(\Gamma_{k}\right)\right)
\end{gathered}
$$

(2) The First Multiple Zagreb index

$$
\begin{aligned}
P M_{1}\left(\Gamma_{k}\right) & =\prod_{u v \in E\left(\Gamma_{k}\right)}[d(u)+d(v)] \\
& =3^{k}(2+k)^{k}
\end{aligned}
$$




\section{Available online at www.ijrat.org}

(3) The Second Multiple Zagreb index

$$
\begin{aligned}
P M_{2}\left(\Gamma_{k}\right) & =\prod_{u v \in E\left(\Gamma_{k}\right)} d(u) d(v) \\
& =(2)^{k}(2 k)^{k} \\
& =(2 k)^{2 k} \\
& =E\left(\Gamma_{k}\right)^{E\left(\Gamma_{k}\right)}
\end{aligned}
$$

(4) The First Zagreb Polynomial index

$$
\begin{aligned}
M_{1}\left(\Gamma_{k}, x\right) & =\sum_{u v \in E\left(\Gamma_{k}\right)} x^{[d(u)+d(v)]} \\
& =k x^{2}\left(x+x^{k}\right)
\end{aligned}
$$

(5) The Second Zagreb Polynomial index

$$
\begin{aligned}
M_{1}\left(\Gamma_{k}, x\right) & =\sum_{u v \in E\left(\Gamma_{k}\right)} x^{d(u) d(v)} \\
& =k\left(x^{2}+x^{2 k}\right)
\end{aligned}
$$

Hence (1) - (5) holds. This completes the proof.

Theorem 3.2. Let $\left(\Gamma_{k}\right)$ be the Line Graph of $k$ Gamma Graph, where $k \geq 2$. Then its topological indices are given by

(1) $M_{3} L\left(\Gamma_{k}\right)=2 E_{2}\left(L\left(\Gamma_{k}\right)\right)$

(2) $P M_{1} L\left(\Gamma_{k}\right)=(1+k)^{k}(2 k)^{\frac{k^{2}-k}{2}}$

(3) $P M_{2} L\left(\Gamma_{k}\right)=K^{E\left(L\left(\Gamma_{k}\right)\right)}$

(4) $M_{1} L\left(\Gamma_{k}, x\right)=E_{1}\left(L\left(\Gamma_{k}\right)\right) x^{(1+k)}+E_{2}\left(L\left(\Gamma_{k}\right)\right) x^{2 k}$

(5) $M_{2} L\left(\Gamma_{k}, x\right)=E_{1}\left(L\left(\Gamma_{k}\right)\right) x^{k}+E_{2}\left(L\left(\Gamma_{k}\right)\right) x^{k^{2}}$

Proof.
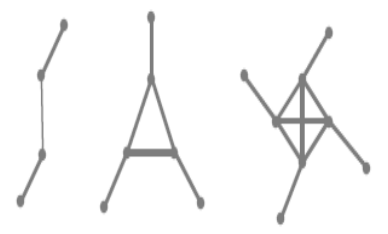

$k=4$

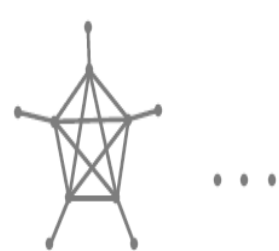

$k=5$
Fig. 3.3. Line Graph of $k$ - Gamma Graph $L\left(\Gamma_{k}\right)$

Consider the Line graph of $k$ - Gamma Graph $L\left(\Gamma_{k}\right)$, for any $k \geq 2$. From the Fig. 3.3., the number of vertices and edges of $L\left(\Gamma_{k}\right)$ is given by $2 k$ vertices and $\frac{k^{2}+k}{2}$ edges. Then the vertex set can be divided into $k$ vertices of degree one and $k$ vertices of degree $k$. Then, the edge set $E\left(L\left(\Gamma_{k}\right)\right)$ can be divided into two edge partitions based on the degrees of its end vertices. The first partition of edges $E_{1}\left(L\left(\Gamma_{k}\right)\right)$ contains $\quad k$ edges $u v, \quad$ where $\quad d_{L\left(\Gamma_{k}\right)}(u)=1$, $d_{L\left(\Gamma_{k}\right)}(v)=k$. and the second partition of edges $E_{2}\left(L\left(\Gamma_{k}\right)\right)$ contains $\frac{k^{2}-k}{2}$ edges $u v$, where $d_{L\left(\Gamma_{k}\right)}(u)=$ $k, d_{L\left(\Gamma_{k}\right)}(v)=k$ Here $d_{L\left(\Gamma_{k}\right)}(u)$ and $d_{L\left(\Gamma_{k}\right)}(v)$ are denoted as the degrees of its end vertices respectively. Now, we shall obtain our required results for the graph $L\left(\Gamma_{k}\right)$ as follows,

(1) The Third Zagreb index

$$
\begin{aligned}
M_{3}\left(L\left(\Gamma_{k}\right)\right) & =\sum_{u v \in E\left(L\left(\Gamma_{k}\right)\right)}|d(u)-d(v)| \\
& =k|k-1|+\frac{k^{2}-k}{2}|k-k| \\
& =k(k-1) \\
& =2 E_{2}\left(L\left(\Gamma_{k}\right)\right)
\end{aligned}
$$

(2) The First Multiple Zagreb index

$$
\begin{aligned}
P M_{1}\left(L\left(\Gamma_{\mathrm{k}}\right)\right) & =\prod_{u v \in E\left(L\left(\Gamma_{k}\right)\right)}[d(u)+d(v)] \\
& =(1+k)^{k}(2 k)^{\frac{k^{2}-k}{2}}
\end{aligned}
$$

(3) The Second Multiple Zagreb index

$$
\begin{aligned}
P_{2}\left(L\left(\Gamma_{\mathrm{k}}\right)\right)= & \prod_{u v \in E\left(L\left(\Gamma_{k}\right)\right)} d(u) d(v) \\
& =k^{\frac{k^{2}+k}{2}} \\
& =k^{E\left(L\left(\Gamma_{k}\right)\right)}
\end{aligned}
$$

(4) The First Zagreb Polynomial index

$$
\begin{aligned}
M_{1}\left(L\left(\Gamma_{k}\right)\right)= & \sum_{u v \in E\left(L\left(\Gamma_{k}\right)\right)} x^{[d(u)+d(v)]} \\
= & k x^{1+k}+\frac{k^{2}-k}{2} x^{2 k} \\
= & E_{1}\left(L\left(\Gamma_{k}\right)\right) x^{(1+k)}+ \\
& E_{2}\left(L\left(\Gamma_{k}\right)\right) x^{2 k}
\end{aligned}
$$

(5) The Second Zagreb Polynomial index

$$
\begin{aligned}
M_{2}\left(L\left(\Gamma_{k}\right)\right)= & \sum_{u v \in E\left(L\left(\Gamma_{k}\right)\right)} x^{d(u) d(v)} \\
= & k x^{k}+\frac{k^{2}-k}{2} x^{k^{2}} \\
= & E_{1}\left(L\left(\Gamma_{k}\right)\right) x^{k}+ \\
& E_{2}\left(L\left(\Gamma_{k}\right)\right) x^{k^{2}}
\end{aligned}
$$

Hence (1)-(5) holds. This completes the proof.

\section{SOME ZAGREB INDICES ON SWASTIKA GRAPH AND ITS LINE GRAPH}

In this section, we can calculate some of the topological indices which are defined earlier for our newly defined $n$ - Swastika Graph $\left(S W_{n}\right)$ and its Line Graph.

Definition 4.1. The four number of Gamma Graphs are joined exactly at their common vertex (starting vertex) is said to be Tetra Gamma Graph or Swastika Graph $\left(\Gamma_{4}\right)$. The degree of Central or Junction vertex Swastika Graph $(S W(G))$ is four.

Definition 4.2. The $n$ - number of Swastika Graph are combined by the Edge Contraction principle [9] at the pendant vertex of each Swastika Graph. The graph 


\section{Available online at www.ijrat.org}

obtained is called $n$ - Swastika Graph $\left(S W_{n}\right)$ for $n \geq 1$. It contains $(7 n+2)$ vertices and $(7 n+1)$ edges.

Theorem 4.1. Let $\left(S W_{n}\right)$ be the $n$ - Swastika Graph, where $n \geq 1$. Then its topological indices are given by (1) $M_{3}\left(S W_{n}\right)=2(5 n+1)$

(2) $P M_{1}\left(S W_{n}\right)=3^{2 V\left(L\left(S W_{n}\right)\right)} 2^{2\left(V\left(\left(S W_{n}\right)\right)-2\right)}$

(3) $P M_{2}\left(S W_{n}\right)=2^{16 n}$

(4) $M_{1}\left(S W_{n}, x\right)=x^{3}\left[2 n\left(2 x^{3}+\frac{x}{2}+1\right)+(2-x)\right]$

(5) $M_{2}\left(S W_{n}, x\right)=x^{2}\left[n\left(2+x^{2}+4 x^{6}\right)+\left(2-x^{2}\right)\right]$

\section{Proof.}
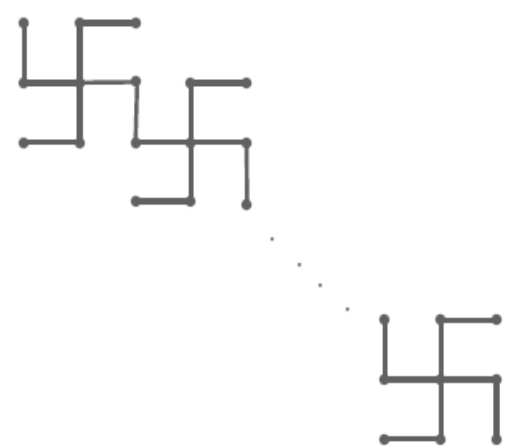

Fig.4.1. $n$ - Swastika Graph $\left(S W_{n}\right)$

Consider the Swastika Graph $S W_{n}$, where $n \geq 1$. From the Fig.4.1, we have $(7 n+2)$ vertices and $(7 n+1)$ edges respectively. Then the vertex set, we can see that there are three vertex partitions such as, $(2 n+1)$ vertices of degree one, $\mathrm{n}$ vertices of degree four and $4 \mathrm{n}$ vertices of degree two. The edge set $E\left(S W_{n}\right)$ can be divided into three edge partitions based on the degrees of its end vertices. The first partition of edges $E_{1}\left(S W_{n}\right)$ contains $(2 n+2)$ edges $u v$, where $d_{S W_{n}}(u)=1, d_{S W_{n}}(v)=2$. The second partition of edges $E_{2}\left(S W_{n}\right)$ contains $(n-1)$ edges $u v$, where $d_{S W_{n}}(u)=2$, $d_{S W_{n}}(v)=2$ and the third partition of edges $E_{3}\left(S W_{n}\right)$ contains $4 n$ edges $u v$, where $d_{S W_{n}}(u)=2, d_{S W_{n}}(v)=4 . \quad$ Here $d_{S W_{n}}(u)$ and $d_{S W_{n}}(v)$ are denoted as the degrees of the end vertices respectively.

Then we shall obtain our required results for the graph $S W_{n}$ as follows,

(1) The Third Zagreb index

$$
\begin{aligned}
M_{3}\left(S W_{n}\right)= & \sum_{u v \in E\left(S W_{n}\right)}|d(u)-d(v)| \\
& =2(5 n+1)
\end{aligned}
$$

(2) The First Multiple Zagreb index

$$
\begin{aligned}
P M_{1}\left(S W_{n}\right) & =\prod_{\substack{u v \in E\left(S W_{n}\right)\\
}}[d(u)+d(v)] \\
& =3^{(2 n+2)} 4^{(n-1)} 6^{4 n}
\end{aligned}
$$

$$
\begin{aligned}
& =3^{2(3 n+1)} 2^{2(3 n-1)} \\
& =3^{2 V\left(L\left(S W_{n}\right)\right)} 2^{2\left(V\left(\left(s W_{n}\right)\right)-2\right)}
\end{aligned}
$$

(3) The Second Multiple Zagreb index

$$
\begin{aligned}
P M_{2}\left(S W_{n}\right) & =\prod_{u v \in E\left(S W_{n}\right)} d(u) d(v) \\
& =2^{(2 n+2)} 4^{(n-1)} 8^{4 n} \\
& =2^{16 n}
\end{aligned}
$$

(4) The First Zagreb Polynomial index

$$
\begin{aligned}
& M_{1}\left(S W_{n}, x\right)=\sum_{u v \in E\left(S W_{n}\right)} x^{[d(u)+d(v)]} \\
& =2(n+1) x^{3}+(n-1) x^{4}+4 n x^{6} \\
& =x^{3}\left[2 n\left(2 x^{3}+\frac{x}{2}+1\right)+(2-x)\right]
\end{aligned}
$$

(5) The Second Zagreb Polynomial index

$$
\begin{aligned}
& M_{2}\left(S W_{n}, x\right)=\sum_{u v \in E\left(S W_{n}\right)} x^{d(u) d(v)} \\
& =2(n+1) x^{2}+(n-1) x^{4}+4 n x^{8} \\
& =x^{2}\left[n\left(2+x^{2}+4 x^{6}\right)+\left(2-x^{2}\right)\right]
\end{aligned}
$$

Hence (1)-(5) holds. This completes the proof.

Theorem 4.1. Let $\left(S W_{n}\right)$ be the Line Graph of $n$ Swastika Graph for every integer $n$. Then its topological indices are given by

(1) $M_{3}\left(L\left(S W_{n}\right)\right)=12 n$

(2) $P M_{1}\left(L\left(S W_{n}\right)\right)=5^{4 n} 8^{6 n}$

(3) $P M_{2}\left(L\left(S W_{n}\right)\right)=2^{32 n}$

(4) $M_{1}\left(L\left(S W_{n}, x\right)\right)=2 n x^{5}\left(3 x^{3}+2\right)$

(5) $M_{2}\left(L\left(S W_{n}, x\right)\right)=2 n x^{4}\left(3 x^{12}+2\right)$

\section{Proof.}
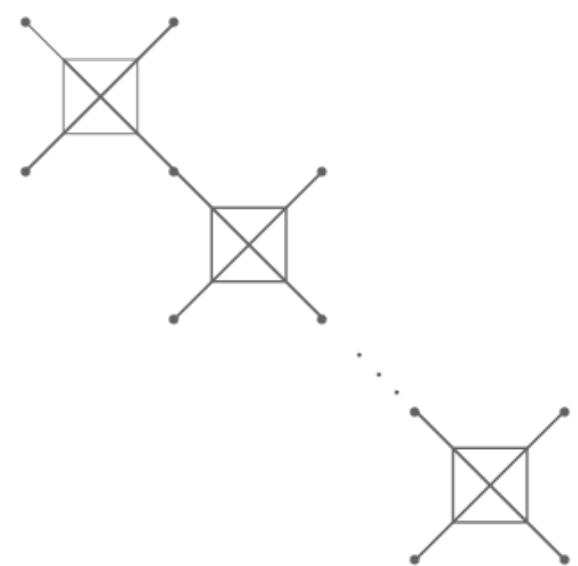

Fig 4.2. Line Graph of $n$ - Swastika Graph $L\left(S W_{n}\right)$

Consider the Line graph of Swastika Graph $L\left(S W_{n}\right)$, for all positive integers $n \geq 1$. From the Fig. 4.2., we get $(7 n+1)$ vertices and $(10 n)$ edges. From the above Fig 4.2., the vertex set can be partitioned into $(3 n+1)$ vertices of degree one and $4 n$ vertices of degree four. Then, the edge set $E\left(L\left(S W_{n}\right)\right)$ can be divided into two edge partitions based on the degrees of its end vertices. The first edge partition 


\section{Available online at www.ijrat.org}

$E_{1}\left(L\left(S W_{n}\right)\right)$ contains $4 n$ edges $u v$, where $d_{L\left(s W_{n}\right)}(u)=1, d_{L\left(s W_{n}\right)}(v)=4$ and the second edge partition of edges $E_{2}\left(L\left(S W_{n}\right)\right)$ contains $6 n$ edges $u v$, where $\quad d_{L\left(s w_{n}\right)}(u)=4, d_{L\left(s W_{n}\right)}(v)=4$. Here $d_{L\left(s W_{n}\right)}(u)$ and $d_{L\left(s W_{n}\right)}(v)$ are denoted as the degrees of the end vertices respectively. Next, we shall obtain our required results for the graph $L\left(S W_{n}\right)$ as follows,

(1) The Third Zagreb index

$$
\begin{aligned}
M_{3}\left(L\left(S W_{n}\right)\right) & =\sum_{\substack{u v \in E\left(L\left(S W_{n}\right)\right)\\
}}|d(u)-d(v)| \\
& 12 n
\end{aligned}
$$

(2) The First Multiple Zagreb index

$$
\begin{aligned}
P M_{1}\left(L\left(S W_{n}\right)\right)= & \prod_{\substack{u\\
}} \prod_{E\left(L\left(S W_{n}\right)\right)}[d(u)+d(v)] \\
& =5^{4 n} 8^{6 n}
\end{aligned}
$$

(3) The Second Multiple Zagreb index

$$
\begin{aligned}
P M_{2}\left(L\left(S W_{n}\right)\right)= & \prod_{u v \in E\left(L\left(S W_{n}\right)\right)} d(u) d(v) \\
= & (4)^{4 n}(16)^{6 n} \\
& =2^{32 n}
\end{aligned}
$$

(4) The First Zagreb Polynomial index

$$
\begin{aligned}
M_{1}\left(L\left(S W_{n}, x\right)\right) & =\sum_{u v \in E\left(L\left(S W_{n}\right)\right)} x^{[d(u)+d(v)]} \\
& =4 n x^{5}+6 n x^{8} \\
& =2 n x^{5}\left(3 x^{3}+2\right)
\end{aligned}
$$

(5) The Second Zagreb Polynomial index

$$
\begin{aligned}
M_{2}\left(L\left(S W_{n}, x\right)\right) & =\sum_{u v \in E\left(L\left(S W_{n}\right)\right)} x^{d(u) d(v)} \\
& =4 n x^{4}+6 n x^{16} \\
& =2 n x^{4}\left(3 x^{12}+2\right)
\end{aligned}
$$

Hence (1)-(5) holds. This completes the proof.

Remark 4.1. For any $k$ - Gamma Graph, the degree of the vertex is always less than or equal to $k$ i.e. $d(u) \leq k$.

\section{CONCLUSION}

In this paper, we calculated some of the Zagreb types of topological indices for our newly defined $k$ Gamma Graph $\left(\Gamma_{k}\right), k$ - Gamma Line Graph $L\left(\Gamma_{k}\right), n$ Swastika Graph $\left(S W_{n}\right)$ and $n$ - Swastika Line Graph $L\left(S W_{n}\right)$. The properties of certain newly defined molecular graphs will be helpful for further studies in drug discovery system.

\section{REFERENCES}

[1] Adnan Aslam; Juan Luis Garcia Guirao; SafyanAhmnad and Wei Gao. (2017): Topological indices of the Line Graph of Subdivision Graph of Complete Bipartite Graphs, Appl. Math. Inf. Sci., 11, (6), pp. 1631-1636.

[2] Gutman, I. ; Das K. C. ,(2004): The First Zagreb Index 30 Years After, MATCH Commun.Math.Comput. Chem.50, pp.83-92.

[3] Fath-Tabar, G. H. (2011): Old and New Zagreb Indices of Graphs. MATCH Commun. Math. Comput. Chem., 65(1), pp.7984.

[4] Hafiz Mutee Ur Rehman; RiffatSardar; Ali raza.(2017): Computing Topological Indices of Hex Board and its line graph, Open J. Math.Sci.,1(1) pp.62 - 71.

[5] ModjtabaGhorbani; NasrinAzimi(2012): Note on multiple Zagreb Indices Iranian Journal of Mathematical Chemistry3(2),pp.137-143.

[6] Mohammed Reza Farahani (2014): First and Second Zagreb Polynomials of $V C_{5} C_{7}[p, q]$ and $\mathrm{HC}_{5} \mathrm{C}_{7}[p, q]$ nanotubes, International Letters of Chemistry Physics and astronomy, 31, pp.56-62.

[7] Venkatachalapathy, M. ; , Kokila, K.; Abarn,B.(2016): Some Trends In Line Graphs, Advances in Theoretical and Applied Mathematics, 11(2), pp.171-178.

[8] Wei Gao; Mohamad Nazri Husin; Mohammad Reza Farahani; Muhammad Imran. (2016): On the Edges Version of Atom-Bond Connectivity and Geometric Arithmetic Indices of Nanocones

CNCkn, Journal of Computational and Theoretical Nanoscience, 13 (10), pp, 67416746.

[9] Wolle, T. ; Bodlaender, H. L. (2004): A Note on Edge Contraction. Technical Report UU-CS2004028, Institute of Information and Computing Sciences, Utrecht University, Utrecht, the Netherland. 